\title{
Determination of neutron spectrum outside the lateral shielding of Taiwan Photon Source using high-sensitivity Bonner cylinders
}

\author{
Yu-Chi Lin ${ }^{1,2}$, Ang-Yu Chen ${ }^{1}$, Joseph Liu ${ }^{1}$, Kuo-Wei Lee ${ }^{2}$, Rong-Jiun Sheur,3,a
}

${ }^{1}$ National Synchrotron Radiation Research Center, 101 Hsin-Ann Road, Hsinchu Science Park, Hsinchu, Taiwan
${ }^{2}$ Institute of Nuclear Engineering and Science, National Tsing Hua University, 101, Sec. 2, Kuang-Fu Road, Hsinchu, Taiwan
${ }^{3}$ Department of Engineering and System Science, National Tsing Hua University, 101, Sec. 2, Kuang-Fu Road, Hsinchu, Taiwan

\begin{abstract}
A homemade Bonner cylinder spectrometer was used to determine the neutron spectrum from thermal energy up to several $\mathrm{GeV}$ at a heavily-shielded light source facility. The spectrometer, similar to the design of Bonner spheres, features high sensitivity of neutron detection because of using a long cylindrical ${ }^{3} \mathrm{He}$ proportional counter. The spectrum measurement was performed during the facility commissioning by intentionally parking the injected electrons at the septum of the storage ring. Based on a high-fidelity FLUKA simulation, the predicted neutron spectrum at the location of measurement was adopted as the initial guess of spectrum unfolding. The unfolded result indicated an underestimation of the calculated neutron spectrum in the high-energy portion, leading to a substantial revision of the neutron dose rate at the location.
\end{abstract}

\section{Introduction}

Taiwan Photo Source (TPS), situated at the National Synchrotron Radiation Research Center in Hsinchu, is a 3 $\mathrm{GeV}$ light source facility with a circumference of $518 \mathrm{~m}$, aiming to provide synchrotron light with extremely high brilliance and low emittance [1]. Electrons are generated and pre-accelerated to $150 \mathrm{MeV}$ through a linear accelerator (LINAC), with a typical beam output of 2.25 $\mathrm{W}$ corresponding to $5 \mathrm{nC}$ per pulse and $3 \mathrm{~Hz}$ repetition rate. These electrons are further accelerated to $3 \mathrm{GeV}$ in a booster synchrotron and then injected into a storage ring. Fig. 1 shows a schematic layout of the main accelerator components of TPS and the bulk concrete structure. The storage ring and concentric booster were installed in a shared tunnel made of $1 \mathrm{~m}$ thick concrete walls, and the shielding in the injection area and ratchet end walls were enhanced to $1.2 \mathrm{~m}$ thick concrete against injection beam loss and forward-peaked bremsstrahlung, respectively.

According to the facility safety analysis report [2], the dose contributions from the neutron and gamma-ray components outside the lateral wall of the TPS tunnel are roughly comparable. In addition, neutrons with energies higher than $10 \mathrm{MeV}$ contribute more than $50 \%$ of the total neutron dose. These estimates were obtained based solely on Monte Carlo simulations. Because of the detector limitation, conventional neutron dosemeters show almost no response to those high-energy neutrons and the extended-range neutron detectors should be used in this environment for proper dose responses. How accurate are the simulation results? Various factors may affect their accuracies, including beam loss assumptions, geometry and material modelling, cross section data and underlying physics models. Therefore, experimentally determining the neutron energy distribution outside the accelerator tunnel is of great interest, in particular the high-energy portion of the spectrum.

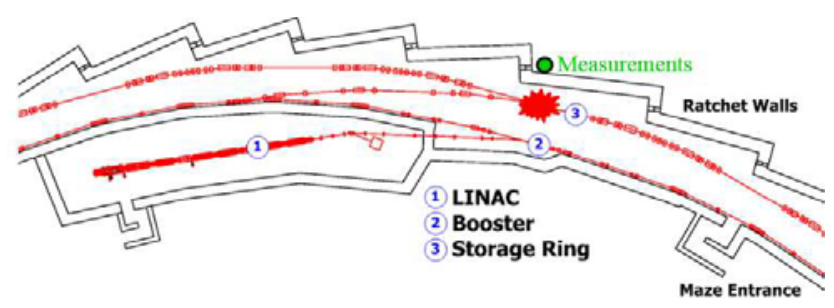

Fig. 1. A schematic layout of the TPS facility, showing the main accelerator components and the bulk concrete structure as well as the location of measurement (green spot).

\section{Materials and methods}

\subsection{Spectrometer and experimental setup}

Bonner spheres are widely used in neutron spectrometry because of the advantages of isotropic and wide-energy responses to incoming neutrons. However, the detection efficiency of a Bonner sphere is limited by the small active volume of its central probe and could become impractical in determining neutron spectra of low intensity. Thus, as shown in Fig. 2, a set of Bonner cylinders was assembled by warping polyethylene (PE) of various thicknesses around a long cylindrical ${ }^{3} \mathrm{He}$ proportional counter. The adopted neutron probe had an

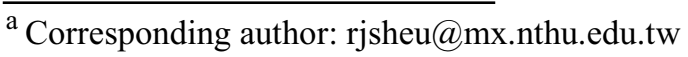


active volume of $2.54 \mathrm{~cm}$ in diameter and $45.72 \mathrm{~cm}$ in length that filled with 4 atm helium-3 gas, exhibiting a sensitivity of $\sim 18$ times higher than the typical probe used in the famous Bonner sphere spectrometer (BSS) NEMUS [3]. The Bonner cylinder spectrometer (BCS) consisted of 16 configurations in total, including the bare tube and 11 combinations with PE cylinders of thicknesses ranging from 1.5 to $10 \mathrm{~cm}$. In addition to these standard configurations, four sets of special cylinders labelled 2C2.5_6.5,2P2.5_6.5, 2P3_6.5, and 3P4_6.5 were the extended-range versions. The naming, taking 2P2.5 6.5 for example, indicated the ${ }^{3} \mathrm{He}$ tube being surrounded by consecutive shells of $2-\mathrm{cm}$-thick PE, $0.5-\mathrm{cm}$-thick lead and another 4-cm-thick PE. The character $\mathrm{P}$ in the naming distinguished the material of the embedded metal in moderator, $\mathrm{P}$ was lead and $\mathrm{C}$ stood for copper.

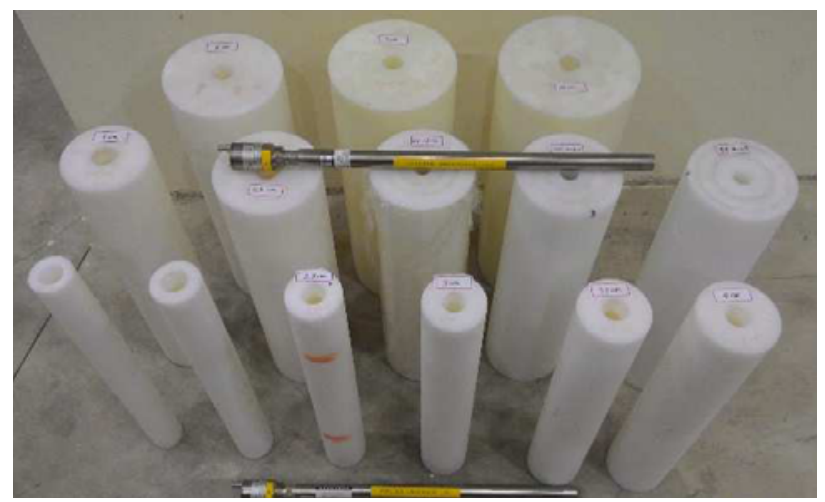

Fig. 2. A photo of the homemade high-sensitivity Bonner cylinders used in this study.

Both Monte Carlo simulations and measurements were performed to investigate the characteristics of the neutron field at the TPS. In order to well define the beam loss location, we intentionally parked all the injected electrons from the booster at the injection septum by turning off the power supply of the magnet. The detectors were arranged at the location outside the lateral shielding wall of the beam loss point, as illustrated in Fig. 1. Because it took time to switch and complete the measurements involving 16 configurations of the spectrometer, a counting rate normalization was necessary to account for the variation of the injected beam. For this reason, an independent neutron detector was installed in a close proximity of the Bonner cylinder to provide a continuous monitoring of the neutron field, which can ensure all the measurements corresponded to the same strength of irradiation. The reference neutron detector was extended-range type and its setup is shown in Fig. 3.

During the experiment, the injected beam current was kept stable and continuously monitored by checking the reading of the booster current. The reading is shown in Fig. 4, indicating an average current of approximately $0.027 \mathrm{~mA}$, corresponding to a beam output of about 40 pC per pulse. Fig. 4 also shows the dose rate history of two nearby neutron and gamma-ray detectors during that period of time. The neutron and gamma-ray dose rates were indeed comparable as expected in the safety analysis report. Note that the level of dose rates was low, only approximately $0.2-0.4 \mu \mathrm{Sv} / \mathrm{h}$, which hinders the use of conventional BSS in neutron spectrum measurement.

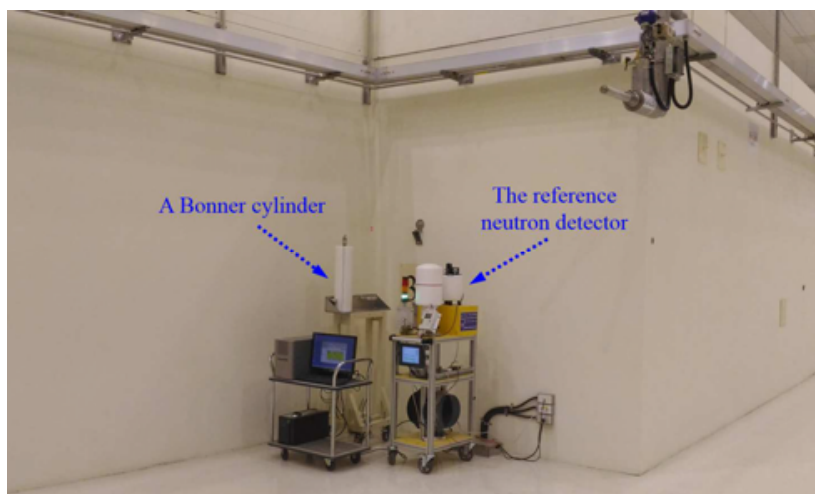

Fig. 3. A photo of the experimental setup at the TPS (See Fig. 1), showing the locations of a Bonner cylinder and the reference neutron detector.

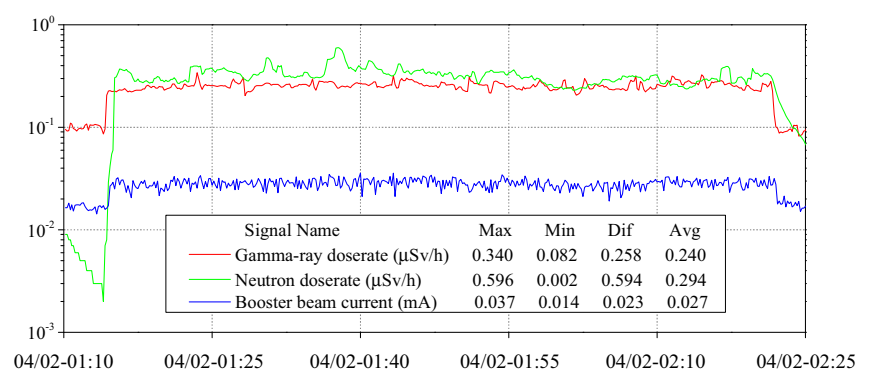

Fig. 4. Monitoring of the booster beam current during the experiment and the dose rate history of two nearby detectors.

\subsection{Monte Carlo simulations}

Two types of Monte Carlo simulations were conducted to provide information regarding the response functions of detectors and the characteristics of radiation field at the TPS. The response functions of 16 configurations of the BCS were calculated using MCNPX [4] with evaluated cross-section data for neutron energies below $150 \mathrm{MeV}$ and the Bertini nuclear model for neutron interactions above $150 \mathrm{MeV}$. Full details of the neutron probe and moderator configurations were included in the calculation model. The resulting response functions were checked and verified by a series of integral tests performed in a standard calibration room using Cf-252 neutron source. For more details on the calculation model and verification of response functions, interested readers are referred to the paper [5].

Radiation environment outside thick shielding of a high-energy electron accelerator mainly consists of bremsstrahlung and secondary neutrons, resulting from the dominant electromagnetic cascade and subsequent photonuclear reactions, respectively. To acquire a quantitative description of the radiation field at the location of measurement, the multi-particle Monte Carlo transport code FLUKA [6] was employed to simulate the shower development and radiation transport around the beam loss spot. A rather detailed geometry model of the TPS injection section, including main components of the 
accelerator system and the surrounding shielding structure, was built aiming to make the simulation as realistic as possible. In addition to dose maps in the region, gamma-ray and neutron spectra were scored at the location of interest. By folding the neutron spectrum with detector response functions, we could predict the neutron counting rates of 16 Bonner cylinders at the location, which can be directly compared with the measurements.

\section{Results and discussion}

\subsection{Detector response functions}

Fig. 5 shows the calculated response functions of 16 Bonner cylinders under a parallel-beam irradiation of neutrons moving perpendicular to the tube axis. The response matrix indicates that the bare tube is essentially sensitive to thermal neutrons only. The 11 standard configurations comprised of PE in various thicknesses can detect neutrons in a wide range of intermediate energies up to several $\mathrm{MeV}$. In contrast, the four extended-range detectors exhibit improved detection efficiencies to high-energy neutrons above $10 \mathrm{MeV}$ due to a neutron multiplication effect caused by the embedded heavy metal. Compared with a conventional BSS, the main feature of this BCS was its high sensitivity, as indicated by the overall magnitude of these response functions. It was essential for our purpose of determining the neutron spectrum at a location with low intensity.

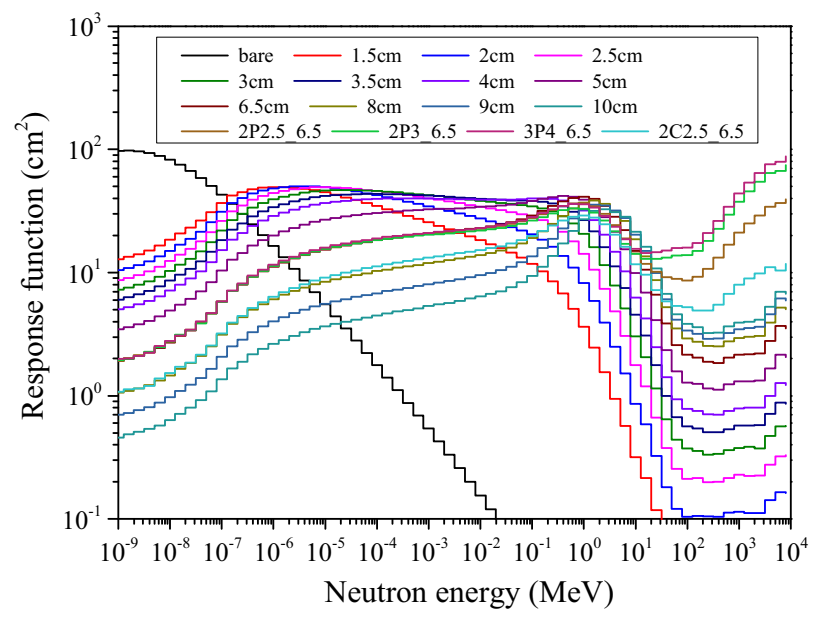

Fig. 5. The MCNPX-calculated response functions of 16 Bonner cylinders for a parallel-beam irradiation.

A problem of the BCS was the loss of an isotropic response to incident neutrons because the cylindricalshaped detectors are not symmetric in the polar angle with respect to the tube axis. The authors prepared another set of response matrix to explore the effect of this problem on spectrum unfolding. In contrast to the parallel-beam irradiation, the second response matrix of the BCS was calculated by considering an isotropic irradiation of neutrons. Fig. 6 shows the calculated results. The two response matrices in Figs. 5 and 6 , respectively, have very similar characteristics but with small differences when observing in details. The isotropic response functions are somewhat lower in magnitude than those calculated under parallel broad beam geometry because of the effect of slant incidence.

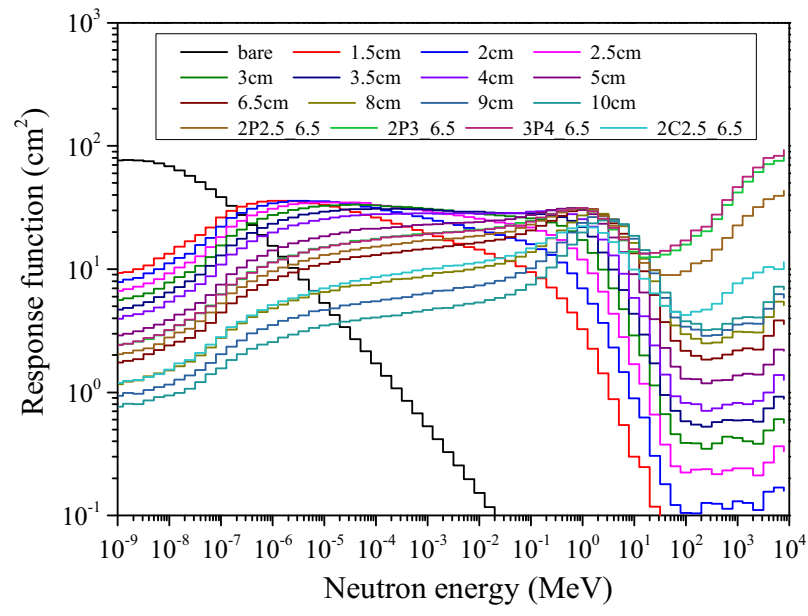

Fig. 6. The MCNPX-calculated response functions of 16 Bonner cylinders for an isotropic irradiation.

\subsection{Characteristics of radiation field}

According to the beam loss scenario and the ICRP-74 ambient dose equivalent conversion factors [7], Fig. 7 shows the FLUKA-predicted neutron and gamma-ray dose distributions around the injection area. In the TPS tunnel, the gamma-ray doses were apparently higher than neutron doses. However, neutrons are generally more penetrating than gamma rays. Outside the lateral shielding wall, neutron doses were observed comparable to or even higher than gamma-ray doses. Note that gamma rays induced by high-energy electrons are significantly forward-peaked, but neutrons lack of a strong directional dependence in this case.
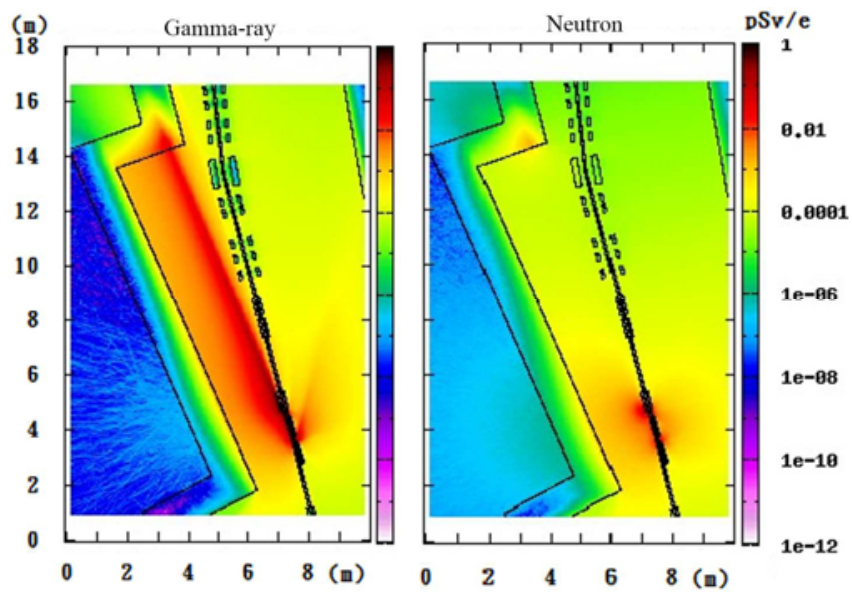

Fig. 7. The FLUKA-predicted neutron and gamma-ray dose distributions around the beam loss point, in unit of pSv per electron loss.

The neutron and gamma-ray spectra at the location of measurement were of particular interest because the information was important to the detector response and dose assessment. Fig. 8 shows the FLUKA-calculated results, where a multiplication factor of 10 was applied for the neutron spectrum to fit in the same scale with the 
gamma-ray spectrum. The energies of most gamma rays are between 0.1 and $10 \mathrm{MeV}$, while the energies of neutrons have a wide range from thermal up to several hundreds of $\mathrm{MeV}$. In addition, high energy neutrons account for an appreciate portion of the total neutrons. Considering their high values of fluence-to-dose conversion coefficients, those abundant high-energy neutrons may cause significant and even higher neutron doses than gamma rays.

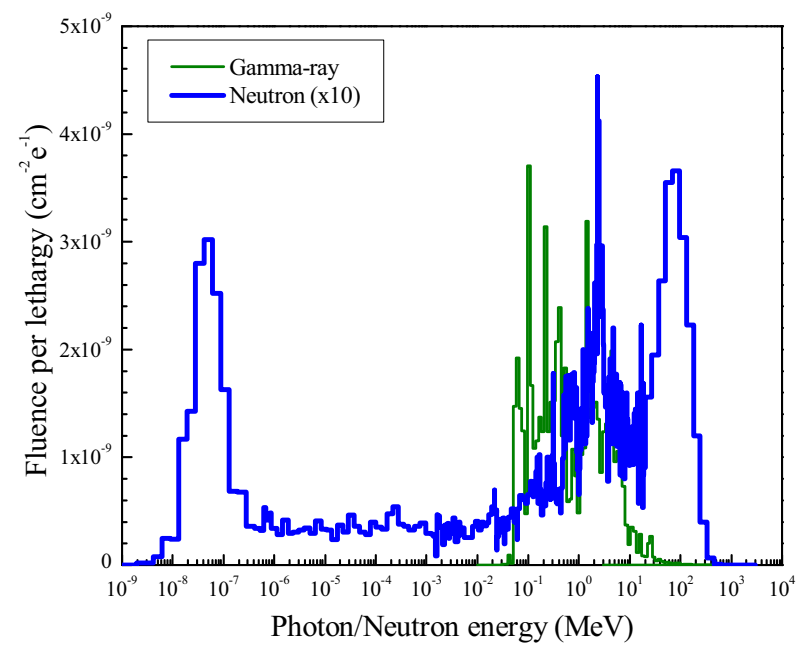

Fig. 8. Neutron and gamma-ray energy spectra scored at the location of measurement.

\subsection{Measurements and spectrum unfolding}

Fig. 9 summarizes the measured neutron counting rates of 16 Bonner cylinders, along with two sets of the FLUKA prediction derived by folding the calculated neutron spectrum with the response functions of detectors. The measured counting rates ranged from approximately 6 to 13 cps depending on the configuration of neutron moderator and material of neutron multiplier. These counting rates were not high and therefore the associated counting fluctuations were obvious because of limited time available for this experiment. On the other hand, the FLUKA-predicted neutron counting rates to some extent agree with the measurements, which in turn strongly indicates that both the Monte Carlo simulations and neutron measurements conducted in this study were reliable because the two approaches share similar trend in an absolute scale. Among these measurements, the counting rates of three lead-embedded detectors (2P2.5_6.5, 2P3_6.5, and 3P4_6.5) were the highest, suggesting a significant percentage of high-energy neutrons presented in the radiation field at the location of measurement.

For an underdetermined problem such as spectrum unfolding, an appropriate initial guess is requisite for most unfolding algorithms. Our best guess was the neutron spectrum in Fig. 8 obtained from the FLUKA simulation. The MAXED deconvolution code [8] based on the maximum entropy principle was used for spectrum unfolding. Because we are not sure of the angular distribution of neutrons incident on the detector, two sets of response matrices in Figs. 5 and 6, respectively, were considered in the unfolding process. As shown in Fig. 10, two unfolded spectra are similar to each other for neutron energies below $10 \mathrm{MeV}$, and without surprise consistent with the FLUKA-predicted result. However, the highenergy neutron peak around $100 \mathrm{MeV}$ was substantially revised by the unfolding code for better agreement with measurements.

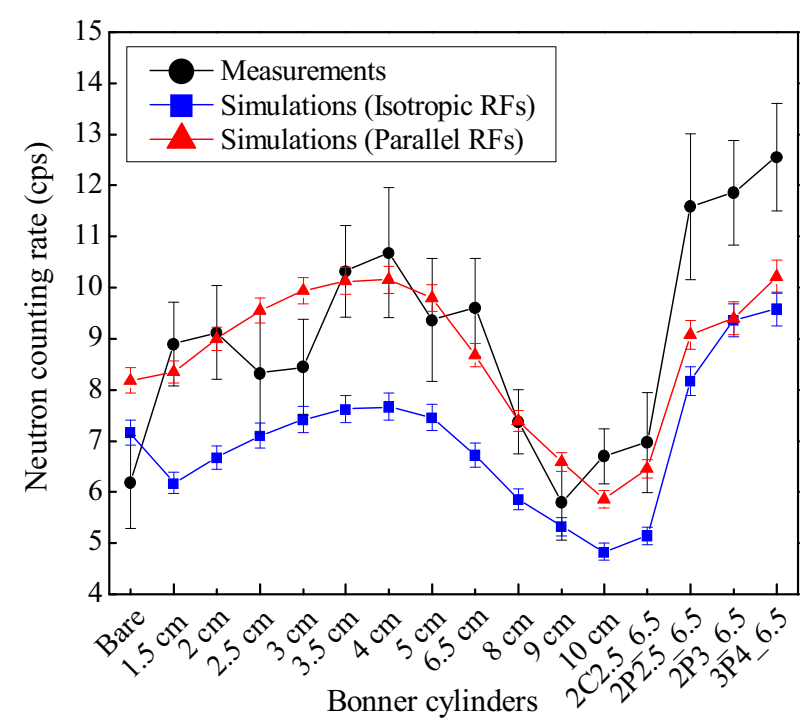

Fig. 9. The FLUKA-predicted neutron counting rates of all Bonner cylinders (with parallel and isotropic response functions) and the measured data.

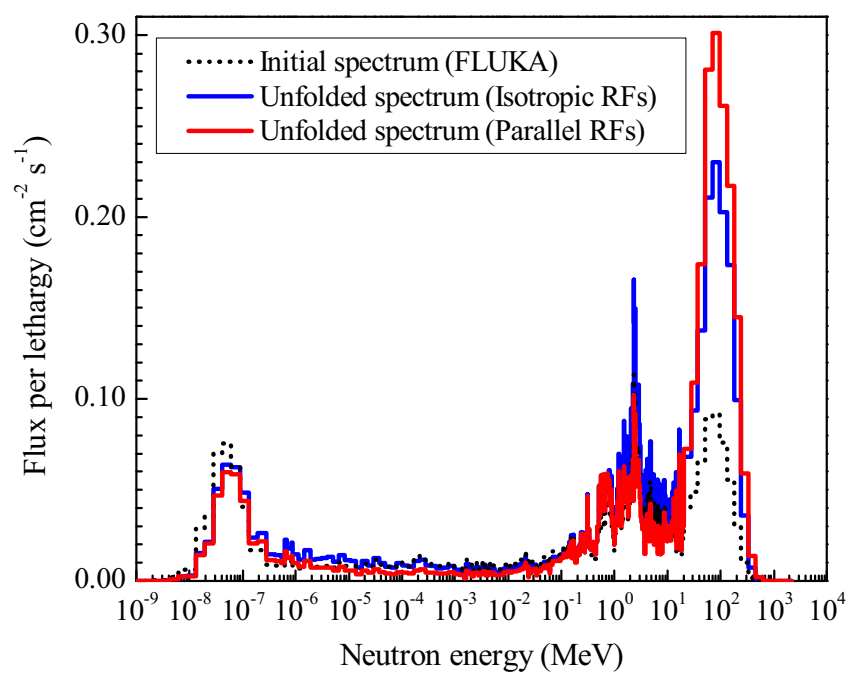

Fig. 10. The FLUKA-calculated initial spectrum and two unfolded spectra based on measurements.

The revision of the high-energy peak in the neutron spectrum has a significant impact on the dose assessment. For the parallel-beam case, the percentage of high-energy neutrons was increased to approximately $61 \%$ of the total neutron flux compared with the initial estimation of $30 \%$ in the calculated spectrum at the location. Taking the fluence-to-dose conversion coefficients into account, the neutron dose rate at the location was estimated to be 0.91 $\mu \mathrm{Sv} / \mathrm{h}$, which was about 2 times higher than the FLUKApredicted dose rate of $0.46 \mu \mathrm{Sv} / \mathrm{h}$ because of a significant 
revision of the neutron spectrum in the high-energy portion. Assuming an isotropic irradiation, as indicated by the comparison of two unfolded spectra in Fig. 10, the high-energy peak was also revised by increasing its importance to better meet the measured data as the parallel-beam case. But the degree of the revision was less than the previous case. High-energy neutrons then accounted for approximately $48 \%$ of the total neutron flux and the neutron dose rate at the location was estimated to be $0.85 \mu \mathrm{Sv} / \mathrm{h}$, still significantly higher than the FLUKA prediction.

\section{Conclusions}

A high-sensitivity BCS was assembled, studied, and tested at the TPS, aiming to determine the neutron spectrum outside the lateral shielding wall of a $3-\mathrm{GeV}$ electron accelerator. For a controlled beam loss scenario, FLUKA was used to simulate the radiation field around the experimental area and calculate the neutron spectrum at the location of measurement. Neutron counting rates of all Bonner cylinders were evaluated by folding the resultant spectrum with corresponding detector response functions. Overall, the calculated results were in satisfactory agreement with the measurements, indicating the response functions of detectors, the characteristics of radiation field, and the measured data were reasonable and reliable.

Regarding the radiation field outside the lateral shielding wall of the TPS, high-energy neutrons are important and dominate the dose contribution. The FLUKA simulation predicted those high-energy neutrons contribute approximately $55 \%$ of the total neutron dose. The unfolded neutron spectrum based on the BCS measurements revised the estimation by raising the percentage of high energy neutrons to $78 \%$ if the parallelbeam response functions were used and $67 \%$ if the isotropic response functions were considered. Both cases suggested an overall correction of the total neutron dose rate by almost doubling the FLUKA prediction. The authors currently are not sure which case can better suit for the actual measuring environment; however, it is reasonable if the answer lies in between these two desperate cases.

\section{Acknowledgments}

The authors would like to thank Yi-Chih Liu for his great help in accelerator operation during measurements. This work was supported by the Ministry of Science and Technology in Taiwan (MOST 104-2623-E-007-004NU).

\section{References}

1. National Synchrotron Radiation Research Center, Taiwan Photon Source design handbook (2009)

2. R.J. Sheu, J. Liu, J.P. Wang, K.K. Lin, G.H. Luo, Nucl. Technol. 168, 417 (2009)
3. B. Wiegel, A.V. Alevera, Nucl. Instrum. Meth. A 476, 52 (2002)

4. D.B. Pelowitz, Ed., MCNPX user's manual, version 2.7.0, LA-CP-11-00438 (2011)

5. K.W. Lee, M.C. Yuan, S.H. Jiang, R.J. Sheu, Radiat. Prot. Dosim. 161, 233 (2014)

6. A. Fasso', A. Ferrari, J. Ranft, P.R. Sala, FLUKA: a multi-particle transport code, CERN-2005-10, INFN/TC_05/11, SLAC-R-773 (2005)

7. ICRP Publication 74, Conversion coefficients for use in radiological protection against external radiation (Pergamon Press, 1996)

8. M. Reginatto, P. Goldhagen, Health Phys. 77, 579 (1999) 